\title{
Three-Component Theory for the Approach to Sedimentation Equilibrium in a Density Gradient
}

\author{
JOHN E. HEARST, Department of Chemistry, University of California, \\ Berkeley, California
}

\section{Synopsis}

A three-component theory for the approach to sedimentation equilibrium in a density gradient is developed starting from the phenomenological equations of irreversible thermodynamics. It is concluded that in the limit of infinitely dilute macromolecule, the properties of the solvated molecule are being measured as might be expected.

\section{Introduction}

Meselson and Nazarian ${ }^{1}$ have presented the equations for the approach to sedimentation equilibrium in a density gradient neglecting the selective solvation of the macromolecule. The purpose of this work is to generalize their result to the three-component case starting with the phenomenological equations of irreversible thermodynamies.

The approximations required for these calculations are similar to those used by Hearst and Vinograd in their equilibrium theory. ${ }^{2}$ A Taylor expansion about the equilibrium position of the band is made for all cell variables and only first-order terms in the displacement parameter retained. Such an assumption limits the use of the theory to short liquid columns but simultaneously eliminates the computational problems associated with a radial field and a sector-shaped cell. The salt gradient is assumed to be at equilibrium at zero time.

The simplicity of the equations is a testament to the ease with which this particular thrce-component system can be understood in the limit of infinitely dilute macromolecule. The introduction of ionic components, which will be considered in a future publication, does not significantly complicate the basic equations derived here.

\section{Theory}

Neglecting Coriolis forces, the phenomenological equations for a threecomponent system in a centrifugal field are: $:^{3,4}$

$$
\begin{aligned}
& J_{1}=L_{11} M_{1}\left(1-\bar{\nu}_{1} \rho\right) \omega^{2} r+L_{13} M_{3}\left(1-\bar{\nu}_{3} \rho\right) \omega^{2} \gamma \\
&-D_{11}\left(d c_{1} / d r\right)-D_{13}\left(d c_{3} / d r\right)
\end{aligned}
$$




$$
\begin{aligned}
J_{3}=L_{33} M_{3}\left(1-\bar{v}_{3} \rho\right) \omega^{2} r & +L_{31} M_{1}\left(1-\bar{v}_{1} \rho\right) \omega^{2} r \\
& -D_{33}\left(d c_{3} / d r\right)-D_{31}\left(d c_{1} / d r\right)
\end{aligned}
$$

The $M_{i}$ are molecular weights and the $\bar{v}_{i}$ are partial specific volumes, where the subscript 1 refers to water and the subscript 3 refers to the macromolecule. This convention is consistent with past publications of the author. The symbols $\omega, r$, and $\rho$ are angular velocity, distance from the center of rotation, and solution density, respectively. The $J_{i}$ represent the molar flux of component $i$ relative to the centrifuge cell. The $c_{i}$ are concentrations in moles per unit volume. The phenomenological coefficients, $L_{i j}$, are related to the diffusion coefficients, $D_{i j}$, by eq. (3)

$$
D_{i j}=\sum_{k} L_{i k} \mu_{k j} \quad(k=1,3)
$$

where

$$
\mu_{k j}=\left(\partial \mu_{k} / \partial c_{j}\right)_{T, P, c k}
$$

Multiplying eq. (1) by $-\left(D_{31} / D_{11}\right)$ and adding to eq. (2) results in eq. (4).

$$
\begin{aligned}
& J_{3}-\frac{D_{31}}{D_{11}} J_{1}=\left(D_{33}-\frac{D_{13} D_{31}}{D_{11}}\right)\left[M_{3}\left(1+\Gamma^{\prime}\right)\left(\frac{\partial \mu_{3}}{\partial c_{3}}\right)_{\mu_{1}}^{-1} \times\right. \\
&\left.\left(1-\frac{\bar{z}_{3}+\Gamma^{\prime} \bar{\nu}_{1}}{1+\Gamma^{\prime}} \rho\right) \omega^{2} r-\frac{d c_{3}}{d r}\right]
\end{aligned}
$$

Equation (3) was used to eliminate the phenomenological coefficients from eq. (4). The solvation parameter, $\Gamma^{\prime}$, is equal to $-\left[\left(M_{1} / M_{3}\right)-\right.$ $\left.\left(\partial \mu_{3} / \partial c_{1}\right)_{c_{3}}\right] /\left(\partial \mu_{1} / \partial c_{1}\right)_{c_{3}}$ which is equivalent to the similar definition of Hearst and Vinograd ${ }^{2}$ based on molalities in the limit of infinitely dilute component 3 .

In the limit of infinitely dilute macromolecule, $D_{31} \rightarrow 0$ while $D_{33}, D_{11}$, and $D_{13}$ remain finite. ${ }^{4}$ This is necessary in order that the flux of component 3 go to zero when the concentration of component 3 is zero. In addition $J_{1}$ is of order $c_{3}$. Therefore to first order in $c_{3}$ we may write:

$$
J_{3}=D_{33} M_{s}\left(\partial \mu_{3} / \partial c_{3}\right) \mu_{1}^{-1}\left(1-\bar{\nu}_{s} \rho\right) \omega^{2} r-D_{33}\left(d c_{3} / d r\right)
$$

where

$$
M_{s}=M_{3}\left(1+\Gamma^{\prime}\right)
$$

and

$$
\bar{v}_{s}=\left(\bar{v}_{3}+\Gamma^{\prime} \bar{v}_{1}\right) /\left(1+\Gamma^{\prime}\right)
$$

At equilibrium $J_{3}=0$, and band position is defined by the point where $\left(d c_{3} / d r\right)=0$. This position we denote $r_{0}$, and the deviation from this position, $r-r_{0}$, is called $\delta$. If all cell variables are expanded about $r_{0}$ and then substituted into eq. (5) with the retention of first-order terms in $\delta$, eq. (6) is obtained. The subscript zero refers to the value of the variable at 
$r_{0}$. $\quad D_{0}$ is $D_{33}$ at $r_{0}$. The gradient in polymer concentration, $d c_{3} / d r$, is of order $\delta$. This fact eliminates the higher order term in the expansion of $D_{33}$.

$J_{3}=\left(D_{0} c_{3} / R T\right) M_{s, 0} \delta\left[\bar{\nu}_{s, 0}(d \rho / d r)+\rho_{0}\left(d \bar{\nu}_{s} / d r\right)\right] \omega^{2} r_{0}-D_{0}\left(d c_{3} / d r\right)$

The substitution

$$
\left(\partial \mu_{3} / \partial c_{3}\right) \mu_{1}=R T / c_{3}
$$

has been made in eq. (6). The validity of this ideality assumption has been discussed previously. ${ }^{2}$ Because the effective gradient originally introduced $^{2}$ is defined by the relation

$$
(d \rho / d r)_{\mathrm{eff}}=(d \rho / d r)+\left(\rho_{0} / \bar{v}_{s, 0}\right)\left(d \bar{v}_{s} / d r\right)
$$

cq. (6) may be further simplified by writing

$$
J_{3}=-\left(D_{0} / \sigma^{2}\right) c_{3} \delta-D_{0}\left(d c_{3} / d \delta\right)
$$

where

$$
\sigma^{2}=R T / M_{s, 0} \bar{v}_{s, 0}(d \rho / d r)_{\mathrm{ef} f} \omega^{2} r_{0}
$$

It is shown in the appendix by using the continuity equation that to first order in $\delta / r_{0}$ we may write

$$
\frac{d m_{n}}{d t}=n \int_{a}^{b} J_{3} \delta^{n-1} d \delta
$$

where $a$ and $b$ are the air-solution meniscus and the bottom of the cell. The moment $m_{n}$ is defined by eq. (10).

$$
m_{n}=\int_{a}^{b} c_{3} \delta^{n} d \delta
$$

By employing the method used by Baldwin and Shooter ${ }^{5}$ and Meselson and Nazarian' ${ }^{2}$ a recursion relation is obtained from eqs. (8), (9), and (10) which enables one to calculate the time dependence of various moments of the distribution.

$$
d m_{n} / d t=-n\left(D_{0} / \sigma^{2}\right) m_{n}+D_{0} n(n-1) m_{n-2}
$$

Equation (11) is integrated to yield:

$$
\begin{array}{cc}
m_{0} \equiv 1 & n=0 \\
m_{1}=\left[m_{1}(0)\right] e^{-D_{0} t / \sigma^{2}} & n=1 \\
{\left[m_{2}-m_{2}(\infty)\right]=\left\{\left[m_{2}(0)-m_{2}(\infty)\right] e^{-2 D_{0} t / \sigma^{2}}\right\}} & n=2
\end{array}
$$

where

$$
m_{2}(\infty)=\sigma^{2}
$$


The symbols $m_{1}(0)$ and $m_{2}(0)$ are the first and second moments of the distribution at zero time.

These solutions are identical in form to those presented by Meselson and Nazarian. ${ }^{1}$ It is clear from this treatment that the properties of the solvated species are being measured.

\section{Appendix}

The continuity equation for a force field with cylindrical symmetry is

$$
d r / d t=-(1 / r) d(J r) / d r
$$

Multiplying eq. (15) by $\delta^{n}$ and integrating from $a$ to $b$ we find

$$
\frac{d m_{n}}{d t}=-\int_{a}^{b} \frac{1}{r_{0}+\delta} \frac{d(J r)}{d \delta} \delta^{n} d \delta
$$

If we now expand

$$
\frac{1}{r_{0}+\delta}=\frac{1}{r_{0}}\left[1-\frac{\delta}{r_{0}}+\left(\frac{\delta}{r_{0}}\right)^{2}-\ldots\right]
$$

and integrate by parts, remembering that $J$ is zero at $a$ and at $b$,

$$
d m_{n} / d t=n \int_{a}^{b} J \delta^{n-1} d \delta-\left(1 / r_{0}\right) \int_{a}^{b} J \delta^{n} d \delta+\left(1 / r_{0}{ }^{2}\right) \int_{a}^{b} J \delta^{n+1} d \delta \ldots
$$

Equation (17) is equivalent to eq. (9) to first-order in $\delta / r_{0}$. There is no justification in keeping the higher order terms here since they were not retained in any of the previous discussion.

The author wishes to thank Leonard Peller and Robert Baldwin for helpful discussions. This work was supported in part by Grant GM-11180 from the National Institutes of Health, U. S. Public Health Service.

\section{References}

1. Meselson, M., and G. M. Nazarian, in Ultracentrifuge Analysis in Theory and Experiment, J. W. Williams, Ed., Academic Press, New York, 1963, pp. 131.

2. Hearst, J., and J. Vinograd, Proc. Natl. Acad. Sci., U. S., 47, 999 (1961).

4. Hooyman, G. J., Physica, 22, 761 (1956).

4. Peller, L., J. Phys. Chem., 29, 415 (1958).

5. Baldwin, R. L., and E. M. Shooter, in Ultracentrifuge Analysis in Theory and Experiment, J. W. Williams, Ed., Academic Press, New York, 1963, p. 143.

Received August 11, 1964

Revised October 16, 1964 